\title{
Electrochemical and Computational Studies of Aripiprazole as a Novel Eco-friendly Green Corrosion Inhibitor for Carbon Steel in Aqueous Environment
}

\author{
Mai Mostafa A. Hassan Shanab \\ Department of Chemistry, Science and Humanity Studies College -Prince Sattam Bin Abdul-Aziz \\ University, P.O. Box: 13 Hawtat Bani Tamim 11149, Saudi Arabia \& Forensic and Toxicology Lab., \\ Forensic Medicine, Mansoura, Egypt. \\ E-mail: $\underline{\text { m.hassan@psau.edu.sa }}$
}

Received: 27 June 2021 / Accepted: 2 August 2021 / Published: 10 September 2021

The theoretical and experimental study of the corrosion inhibitor of the Expired Aripiprazole drug on carbon steel (CS) in hydrochloric acid corrosive medium (1M) has been examined by employing chemical and electrochemical methods. The inhibition efficiency was found to improve with the rise of the concentration of inhibitor and explained on the basis of adsorption metal surface. The adsorption effect follows Langmuir adsorption isotherm. In the presence and absence of expired Aripiprazole the percent inhibition efficiency (\% IE) and activation energy $\left(\mathrm{E}_{\mathrm{a}}{ }^{*}\right)$ were calculated. The impact of temperature on corrosion rate was investigated. The morphology of the CS surface was examined using FTIR, AFM, and XPS tests. Results obtain for all methods used are in good agreement. Also, the theoretical calculations were calculated utilizing density functional theory (DFT) and Hartree Fock (HF) method. This electronic molecular parameter of aripiprazole aid to describe the mechanism of prevents CS corrosion. Monte Carlo simulations were also performed to simulate the adsorption of expired aripiprazole drug on iron surface and the results show that ph-N-C=O is the most effective corrosion inhibitor for CS in acidic medium.

Keywords: Expired Aripiprazole drug, CS, HCl, EIS, AFM, XPS, and DFT

\section{FULL TEXT}

(C) 2021 The Authors. Published by ESG (www.electrochemsci.org). This article is an open access article distributed under the terms and conditions of the Creative Commons Attribution license (http://creativecommons.org/licenses/by/4.0/). 